\title{
Erratum
}

\section{The Kähler cone on Calabi-Yau threefolds}

\section{P.M.H. Wilson}

Department of Pure Mathematics, University of Cambridge, 16 Mill Lane, Cambridge CB2 1SB, UK

Invent. Math. 107, 561-584 (1992)

The results of the above paper require slight modification in the light of some inaccuracies in Section 2 concerning birational contractions of Type III (that is, primitive contractions which contract a surface down to a curve). In summary, it is not only elliptic ruled surfaces on the Calabi-Yau threefold which cause the Kähler cone to jump under general deformations, but also conic bundles over an elliptic curve all of whose fibres are line pairs (i.e. when there is an unramified map of degree two from an elliptic ruled surface to the threefold). The main result of the paper is therefore restated as follows.

Main Theorem. Suppose that $\pi: \mathscr{X} \rightarrow B$ is the Kuranishi family of a Calabi-Yau threefold $X$ over a polydisc $B$ in $H^{1}\left(X, T_{X}\right)$. The Kähler cone is invariant in the family if and only if none of the threefolds $X_{b}$ contain either a smooth elliptic ruled surface or a conic bundle over an elliptic curve all of whose fibres are line pairs. More generally, the Kähler cone will be invariant over the dense subset of $b \in B$ for which $X_{b}$ contains no such surfaces, this subset being the complement of at most countably many codimension one submanifolds of $B$.

In the notation of Section 2, let us suppose that $\phi_{D}: X \rightarrow \bar{X}$ is a contraction of Type III contracting an irreducible surface $E$ on $X$ down to a curve $C$ on $\bar{X}$. As pointed out to the author by Prof. Yoshinori Namikawa, it is not true as stated on page 567 that $C$ will have to be generically a curve of $A_{1}$ singularities, since we also have the possibility of $C$ being generically a curve of $A_{2}$ singularities. In this case the exceptional divisor $E$ is not locally irreducible, but it may still be globally irreducible. Under the hypotheses of Theorem 2.2, the deductions we require in order to prove the Main Theorem are that either $X$ contains a $\mathbb{P}^{1}$ which does not deform in $X$, or the curve $C$ is smooth and $E$ is either a $\mathbb{P}^{1}$-bundle over $C$, or a conic bundle over $C$ all of whose fibres are line pairs. A more detailed classification is not needed here and so will not be pursued. To prove these statements, we should avoid use of Lemma 2.3, which is erroneous as it stands. Instead, we use a similar argument to that given at the bottom of p. 568 .

Let $\phi: E \rightarrow C$ denote the induced morphism on $E$. Since $\phi_{D}$ contracts a single numerical class of curves, it follows for any point $P$ on $C$, that the fibre $\phi^{-1}(P)$ has 
at most two components. If $Z$ denotes the reduced fibre over $P$, then either $S \cdot Z=1$ or 2 (where as on p. 568, $S$ denotes a general element in the linear system $|n D-E|$ ). In the former case, we have that $Z$ is a $\mathbb{P}^{1}$ which does not deform in $X$. In the latter case, we deduce as on page 568 that $C$ has embedding dimension of at most two at $P$, and then the argument given on pp. 568-9 gives a contradiction when $X$ is smooth and $P$ is a singular point of $C$. Since $E$ is a conic bundle over $C$, the general fibre is either irreducible or two lines. Let us assume also that any $\mathbb{P}^{1}$ contained in $X$ does deform in $X$. In the former case, we are in the situation considered in Sect. 3, and it follows that $E$ is in fact a $\mathbb{P}^{1}$-bundle over $C$. In the latter case, it follows that $E$ is a conic bundle over $C$ all of whose fibres are line pairs. In this case, the normalization $\tilde{E}$ of $E$ is a $\mathbb{P}^{1}$-bundle over an étale double cover $\tilde{C}$ of $C$.

Except for the cases of $E$ being a $\mathbb{P}^{1}$-bundle over a smooth curve $C$ or a conic bundle over $C$ all of whose fibres are line pairs, use of Proposition 3.1 ensures that the face of the Kähler cone defined by the fibres of $E$ is invariant under small deformations. We need therefore only consider these two cases. The former case was dealt with in Sect. 4, where it was seen that the existence of $E$ causes the Kähler cone to jump under a general deformation if and only if $C$ has genus 1 . In the latter case, the genus of $C$ must be positive and we consider the unramified morphism $f: \tilde{E} \rightarrow X$, where $\tilde{E}$ is a $\mathbb{P}^{1}$-bundle over $\tilde{C}$; the key now is to consider the deformations of the morphism. Results of Ran (see Corollary 3.2 of [42], or the proof of Theorem 2.1 from [28]) now ensure that the morphism $f$ deforms precisely over a locus of codimension $\tilde{g}=g(\tilde{C})=h^{1}\left(\mathcal{O}_{\tilde{E}}\left(N_{f}\right)\right)$ in the Kuranishi space for $X$, where $N_{f}$ is the invertible sheaf on $\widetilde{E}$ defined by the exact sequence

$$
0 \rightarrow T_{\tilde{E}} \rightarrow f^{*} T_{X} \rightarrow N_{f} \rightarrow 0
$$

(and hence in our case $N_{f}=\omega_{\tilde{E}}$ ). For this we argue by means of Hodge theory exactly as before to show (cf. also [28, Theorem 2.1]) that the natural map $H^{1}\left(T_{X}\right) \rightarrow H^{1}\left(N_{f}\right)$, obtained as the composite $H^{1}\left(T_{X}\right) \rightarrow H^{1}\left(f_{*} f^{*} T_{X}\right)=$ $H^{1}\left(f^{*} T_{X}\right) \rightarrow H^{1}\left(N_{f}\right)$, is surjective. Given this result, a natural generalization of the arguments from Sect. 4 (which we sketch below) shows that jumping of the Kähler cone occurs if and only if $g(C)=1$.

We start by arguing in an analogous way to the proof of (4.2). Let $S$ denote the versal deformation space for an $A_{2}$ singularity, where $S$ may be regarded as a neighbourhood of the origin in $\mathbb{C}^{2}$, with a discriminant curve $R$. Let $P \in C$ be the point corresponding to a given fibre $Z$ of the conic bundle $E$ over $C ; Z$ in this case is a line pair. With the notation as in (4.2), we have an induced map $\theta: B \times \Delta \rightarrow S$. Observe that $\theta^{-1}(0)$ has codimension at most two in $B \times \Delta$. Moreover, the morphism $f: E \rightarrow X$ deforms precisely over a locus in $B$ of codimension $\tilde{g}$, which is greater than two if $g(C)>1$. If therefore $g(C)>1$ and $\theta^{-1}(0)$ has codimension 1 in $B \times A$, the argument given at the end of the proof of (4.2) shows that for every $b \in B$, the threefold $X_{b}$ contains a deformation of some fibre of $E$ as a line pair. If however $\theta^{-1}(0)$ has codimension 2 , then its projection to $B$ has codimension 1 in $B$. This implies that the map $\theta^{-1}(R) \rightarrow B$ is dominating, and so for every $b \in B$, the threefold $X_{b}$ still contains a deformation of some fibre of $E$. This ensures that the corresponding face of the Kähler cone is invariant under small deformations, as required.

In the case when $g(C)=1$, we know that the morphism $f: \tilde{E} \rightarrow X$ deforms precisely over a locus in $B$ which is smooth of codimension one. We observe in this case that there is a natural isomorphism $H^{1}\left(N_{E / X}\right) \rightarrow H^{1}\left(N_{f}\right)$, induced from the isomorphism $H^{0}\left(\omega_{c}\right) \rightarrow H^{0}\left(\omega_{\tilde{C}}\right)$ (cf. p. 574). Since the map $H^{1}\left(T_{X}\right) \rightarrow H^{1}\left(N_{f}\right)$ is 
non-zero, the same will be true of the map $H^{1}\left(T_{X}\right) \rightarrow H^{1}\left(N_{E / X}\right)$. The argument given at the bottom of p. 574 shows then that the image of a class $\theta \in H^{1}\left(T_{X}\right)$ in $H^{1}\left(N_{E / X}\right)$ is non-zero if and only if its image in $H^{1}\left(N_{Z / X}\right) \cong \mathbb{C}$ is non-zero, where $Z$ is any fibre of $E$ over $C$. Observing that $Z$ is locally a complete intersection on $X$, we deduce, for a 1-parameter deformation of $X$ with Kodaira-Spencer class $\theta$, that this image in $H^{1}\left(N_{Z / X}\right)$ is the first order obstruction to $Z$ extending sideways. The locus in $B$ over which a fibre $Z$ deforms is therefore smooth of codimension 1, and coincides with the locus over which $f$ deforms. In other words, if $Z$ deforms sideways in some small deformation of $X$, it does so as a line pair, and so the individual lines deform. We now extend the infinitessimal deformation argument from pp. 576-7 of the paper to deformations of the morphism $f$. The argument of (4.4) and Ran's result reduces us to proving the non-existence of a 1-parameter deformation $\mathscr{X} \rightarrow \Delta$ of $X=X_{0}$ with respect to which the morphism $f$ deforms only up to order $m-1$, but for which the inclusion of some fibre $Z \cong \mathbb{P}^{1}$ deforms to all orders. As in (4.4), we have infinitessmal deformations $\pi_{r}: X_{r} \rightarrow \Delta_{r}$ for all $r \geqq 0$. If $f$ extends to a morphism $f_{r}: \tilde{E}_{r} \rightarrow X_{r}$ of smooth schemes over $\Delta_{r}$, it follows from the theory developed in [41, Exp. III, Sect. 5], that the obstruction to extending $f_{r}$ to a morphism $f_{r+1}: \tilde{E}_{r+1} \rightarrow X_{r+1}$ of smooth schemes over $\Delta_{r+1}$ lies in $H^{1}\left(\widetilde{E}_{r}, N_{f_{r}}\right)$, where $N_{f_{r}}$ is the normal sheaf to the morphism $f_{r}$ defined from the exact sequence

$$
0 \rightarrow T_{\tilde{E}_{r}} \rightarrow f^{*} T_{X_{r}} \rightarrow N_{f_{r}} \rightarrow 0 .
$$

We now follow the argument in the proof of (4.4) replacing $N_{E_{r} / X_{r}}$ by $N_{f_{r}}$, the sheaf $T_{X_{r}} \mid X_{r-1}$ by $f_{r-1}^{*} T_{X_{r}}$ and $N_{E_{r-1} / X_{r}}$ by the cokernel of $T_{\tilde{E}_{r-1}} \rightarrow g_{r-1}^{*} T_{X_{r}}$ (where $g_{r-1}$ is the composite of $f_{r-1}$ and the closed immersion $X_{r-1} \rightarrow X_{r}$ ). As before we deduce that the non-zero obstruction to deforming to order $m$ the morphism $f_{m-1}$ gives rise to a non-zero obstruction to deforming to order $m$ the morphism (closed immersion) $Z_{m-1} \rightarrow X_{m-1}$, and hence a contradiction. As in (4.4), this shows that the existence on $X$ of a conic bundle over an elliptic curve all of whose fibres are line pairs ensures that the Kähler cone will not be invariant under a general small deformation of $X$.

The rest of the main theorem follows immediately from the above facts and previous arguments.

\section{Additional References}

41. Grothendieck, A.: Revêtements Etales et Groupe Fondamental (SGA1). (Lect. Notes Math., Vol. 224) Berlin Heidelberg New York: Springer 1971

42. Ran, Z:: Hodge theory and deformations of maps. Compon. Math. (to appear) 